\title{
Einfluss korrigierender Kosmetik auf die Lebensqualität von Patienten mit fazialen Hautunregelmäßigkeiten
}

M. Keschawarzi

Effects of Corrective Make-up on Life Quality of Patients with Facial Skin Lesions

\section{Zusammenfassung}

Im Gesicht lokalisierte, für jeden sichtbare Hautveränderungen finden sich häufig in der Bevölkerung. Neben der rein organischen Bedeutung haben diese Veränderungen oft eine nicht zu vernachlässigende Auswirkung auf die Lebensqualität der betroffenen Personen. Kurativ-therapeutische Möglichkeiten sind häufig langwierig und ermöglichen nicht immer eine absolute restitutio ad integrum. Außerdem macht die Rezidivfreudigkeit verschiedener Hautunregelmäßigkeiten seinerseits den ursprünglichen Erfolg wieder zunichte. In diesen Fällen steht die Anwendung von Camouflage-Produkten als Ausweg zur Verfügung. Eine Untersuchung zur Auswirkung fazialer Hautunregelmäßigkeiten auf die Lebensqualität und die Verbesserung derselben unter der Anwendung eines korrigierenden Make-ups (Dermablend ${ }^{\circledR}$ ) zeigt auf, dass faziale Hautunregelmäßigkeiten einen Einfluss auf die Lebensqualität der Patienten haben. Und korrigierende Kosmetik ist eine wertvolle Option für Arzt und Patienten und führt zu einer Verbesserung der Lebensqualität.
Abstract

Visible facial skin lesions are common in population. These changes not only have an organic impact but also an essential impact of life quality. The curative-therapeutical options often are longsome and do not lead always to absolute restitution ad integrum. In addition recidivism of different skin lesions defeat the original success. In this cases camouflage products are an efficient resource. In a study the effects of facial skin lesions on life quality and the improvement of life quality under application of the corrective make-up dermablend ${ }^{\circledR}$ were investigated. The results demonstrate that the use of the corrective make-up leads to an improvement of life quality. Corrective make-up is a valuable option for physicians and patients.

\section{Die Rolle der Haut im äußeren Erscheinungsbild}

Das äußere Erscheinungsbild eines jeden Individuums stellt den stärksten beeinflussenden Faktor unserer sozialen Interaktionen dar. Die Haut hat hier, als äußerlich sichtbares Organ neben den Haaren, den größten Einfluss auf unser Selbstbild, unsere Außenwirkung und die individuelle Lebensqualität.

Ein vitaler, gesunder Mensch weist in den meisten Fällen auch eine gesunde Haut auf, was sich zum Beispiel im Colorit, der Reinheit, der Ebenmäßigkeit ausdrückt. Somit wird auch ver- ständlich, dass in unserer vitalitätsorientierten, gesellschaftlichen Weltsicht ein großer Wert auf ein ästhetisches Erscheinungsbild gelegt wird.

Die Haut ist nicht nur das größte menschliche Organ mit entsprechenden Schutzmechanismen. Vielmehr spiegelt sie auch unsere innere Befindlichkeit und Gesundheit wieder. Wird ihr Aussehen durch sichtbare Veränderungen beeinträchtigt, können bei den Betroffenen durch diese Stigmatisierung ernsthafte psychische Probleme ausgelöst werden. Das Spektrum reicht von Angststörungen mit und ohne sozialen Rückzug über De- 
pressionen bis hin zu Suizidalität. Reaktionen der Außenwelt, angefangen bei bloßem Anstarren bis hin zu Benachteiligung bei der Arbeitssuche [1], tragen zur Verschlimmerung der subjektiven Symptomatik bei. Der Dermatologe behandelt deswegen in seiner Praxis längst nicht mehr nur die rein organischen Symptome, sondern berücksichtigt ebenfalls den Einfluss auf die Lebensqualität. Aus diesem Grund steht an erster Stelle der Therapie bei Patienten mit sichtbaren Hautveränderungen eine fundierte Beratung, in der die Erwartungen der Patienten, die Behandlungsmöglichkeiten sowie das zu erwartende Behandlungsergebnis zu eruieren und abzuwägen sind [2].

\section{Behandlung von Hautunregelmäßigkeiten -}

Berücksichtigung der „Down-Time“

Bei Hautunregelmäßigkeiten handelt es sich nicht um ein Minderheitenproblem. So sind beispielsweise vaskuläre Missbildungen wie Hämangiome und Neoplasien häufig und treten bereits bei $5 \%$ der neugeborenen Kinder auf. Auch Pigmentstörungen aus dem Formenkreis der Hypo- bzw. der Hyperpigmentierung, oder Couperose und Rosacea sind ebenfalls nicht selten. Postoperative Läsionen können auch nicht ganz ausgelassen werden.

Die verfügbaren Behandlungsmethoden solcher Veränderungen haben sich mittlerweile so weit entwickelt, dass eine Vielzahl der o.g. Erkrankungen erfolgreich behandelt werden können. Jedoch können auch modernste Ansätze wie zum Beispiel topische Steroide, oder Photo(chemo)-Therapie bis hin zu modernster Lasertechnologie und/oder chirurgische Maßnahmen nicht immer zu einem kosmetisch zufrieden stellenden Ergebnis führen. So sind zum Beispiel die Ergebnisse bei Vitiligo, Chloasma, Melasma oder kutanen Manifestationen von Gefäßmissbildungen individuell sehr unterschiedlich. Der gewünschte Behandlungserfolg kann in vielen Fällen nicht komplett erreicht werden. Das gleiche gilt für Erkrankungen wie der Erythrose und Couperose. Die Behandlungsergebnisse sind häufig, gerade bei heller Haut eher dürftig und werden letztlich durch die hohe Rezidivhäufigkeit unterlaufen.

Ca. 20\% aller Feuermale lassen sich nicht oder nur gering verbessern. Hier muss den Patienten eine schnelle, zuverlässige und auch einfach anzuwendende Lösung angeboten werden, um ihnen einen Alltag ohne Stigmatisierung zu ermöglichen.

An erster Stelle stehen hier Camouflage-Produkte bzw. korrigierende Kosmetik. In diesem Zusammenhang dürfen auch die so genannten postoperativen und/oder therapieinduzierten temporären Läsionen nicht unerwähnt bleiben. Laserbehandlungen, Dermabrasionen, Peelings, Rhinoplastiken etc. hinterlassen oft Zeichen von Hautrötung und/oder Hämatome. Zur Reduktion der Down-Time des Patienten und zur Gewährleistung eines eventuell benötigten Sonnenschutzes (z. B. nach Lasern) müssen auch hier korrigierende Camouflage-Produkte zum Einsatz kommen.

\section{Moderne korrigierende Kosmetilk - effektiv und zuverlässig}

Die Anforderungen an ein so genanntes „ideales abdeckendes Produkt" sind hoch. Es sollte einfach aufzutragen sein, einen guten und dauerhaften Halt haben. Natürliches Aussehen gewähren und eine hohe Verträglichkeit aufweisen. Das 1981 von dem amerikanischen Dermatologen Dr. Robert Craig entwickelte Produkt Dermablend ${ }^{\circledR}$ stellt eine Camouflage-Option dar, die diesen Anforderungen sehr nahe kommt. In den Vereinigten Staaten gilt es als anerkanntes Präparat und ist die kosmetische Nummer 1 bei Dermatologen und plastischen Chirurgen. Die einfache Anwendung sowie die hervorragende Haltbarkeit und Verträglichkeit auf der Haut sind überzeugend.

Das Auftragen ist unkompliziert und erfolgt in zwei Schritten. Zuerst wird das Make-up mit einem kleinen Spatel entnommen und auf dem Handrücken durch Reiben angewärmt. Die nun leicht verteilbare Creme wird im Anschluss zur Abdeckung der betroffenen Hautareale aufgetragen und an den Außenrändern verstrichen, so dass keine Übergänge zu sehen sind. Danach erfolgt die Fixierung mit dem Puder. Der transparente, mikronisierte Puder verbindet sich auf der Haut mit dem Make-up, ohne dabei die Farbe des Make-ups zu verändern und sorgt damit für die starke Haltbarkeit bis zu 16 Stunden. So aufgetragen, kann Dermablend auch extremen Belastungen wie Schwitzen, Baden oder Reiben standhalten. Diese außergewöhnliche Beständigkeit nachgewiesen werden (L'Oréal; Data on File).

Im Testablauf wurde das Korrektur-Make-up mit dem Fixierpuder in den beschriebenen zwei Schritten aufgetragen und die geschminkte Haut mittels Chromametrie erfasst. Eine zweite Messung erfolgte nach einem 30-minütigen Aufenthalt in der Sauna bei Temperaturen zwischen 40 und 45 Celsius und einer relativen Luftfeuchtigkeit von 44 - 49\%. Unter diesen Voraussetzungen wich der gemessene Wert mit nur 0,56 (Chroma-Meter-Messung) von dem Eingangswert ab.

Auch nach zweimaligen 20-minütigen Aufenthalten in der Badewanne mit 23-32 Celsius warmem, sprudelndem Wasser lag der Differenzwert lediglich bei 0,52.

\section{Weitere Wirkstoffe in Dermablend ${ }^{\circledR}$}

Der Pigmentanteil von 40\% gewährt eine effektive Abdeckung auch gravierender Hautunregelmäßigkeiten, ohne dabei das Gesicht maskenhaft erscheinen zu lassen. Aus acht verschiedenen Schattierungen kann der passende Farbton gewählt bzw. gemischt werden. In einem Vergleich von Dermablend versus einem anderen korrigierenden Make-up zeigte Dermablend eine um 20\% höhere Deckkraft als das Vergleichsprodukt. Gegenüber einem gewöhnlichen Tages-Make-up zeigte sich eine um $90 \%$ höhere Deckkraft. Diese Messungen erfolgten Spectral-Colorimetrisch. Der Lichtschutzfaktor (LSF) 30 sorgt für den nötigen Sonnenschutz zum Beispiel bei Vitiligo-Patienten und während Laserbehandlungen. Um eine gute Verträglichkeit zu gewährleisten, wurde auf Duftstoffe verzichtet und neben Pigmenten lediglich acht weitere Bestandteile eingearbeitet, was deutlich unter der Anzahl anderer herkömmlicher Camouflage-Produkte liegt (Tab.1). Andere weisen im Durchschnitt 20-25 Wirkstoffe auf. 
Tab. 1 Inhaltsstoffe des Korrektur-Make-ups Dermablend ${ }^{\circledR}, 8$ Stoffe + Pigmente

\begin{tabular}{l}
\hline INCI \\
\hline Paraffinum Liquidum \\
\hline Cera Alba \\
\hline Talcum \\
\hline Isopropyl Palmitat \\
\hline Magnesiumcarbonat \\
\hline Carnauba \\
\hline Propylparaben \\
\hline Allantoin \\
\hline Titandioxid/Cl 77891 \\
\hline Eisen Oxid/Cl $77491, \mathrm{Cl} 77499, \mathrm{Cl} 77492$ \\
\hline
\end{tabular}

Weitere Inhaltsstoffe von Dermablend ${ }^{\circledR}$ sind Carnauba- und Bienenwachs. Diese dienen der Pflege der Haut und sorgen für einen verbesserten Feuchtigkeitszustand. Allantoin wirkt Haut beruhigend. Das Abschminken erfolgt mit einem normalen Make-up Entferner.

\section{Quality of Life und Camouflage -}

klinische Untersuchungsergebnisse

Es besteht große Einigkeit, dass „Mortalität“ nicht der einzige wichtige Effekt einer Hauterkrankung ist. Die Lebensqualität oder auch „Quality of life“ (QOL) spielt hier eine mindestens ebenso große Rolle. Die „QOL“ umfasst physische, soziale, psychologische Aspekte sowie die täglich empfundene Belastung des betroffenen Individuums. Die Messung des „QOL“-Komplements lässt seinerseits Rückschlüsse auf die Krankheitsaktivität $\mathrm{zu}[3]$.

Für die Erfassung der „QOL“ gibt es verschiedene Arten von Fragebogen: so genannte übergreifende Fragebogen und solche, die eher organ-/krankheitsspezifisch aufgezogen sind. Die übergreifenden Fragebogen dienen der Erfassung des Einflusses der Erkrankung auf die Tagesaktivität und die allgemeine Funktionsfähigkeit. Sie werden eingesetzt, um verschiedene Bedingungen miteinander zu vergleichen und sind relativ unsensibel für Hauterkrankungen. Organ- bzw. krankheitsspezifische Fragebogen sind hier sehr viel sensibler für die Erfassung von krankheitsspezifischen Auswirkungen einer vorgegebenen Erkrankung. Sie sind allerdings nicht gut geeignet für den Vergleich der Einflüsse verschiedener Erkrankungen.

In einer Studie zum Einfluss korrigierender Kosmetik auf die Lebensqualität von Patienten mit stigmatisierenden Hauterkrankungen wurden drei verschiedene „QOL“-Fragebogen eingesetzt:

1. Skindex-16 Fragebogen

2. Modifizierter „Dermatology Life quality Index“ (DLQI),

3. „Fear of negative evaluation“ (FNE) Fragebogen.
Tab. 2 Häufigkeit der in der Studie aufgenommenen Hautveränderungen

\begin{tabular}{|lr}
\hline Diagnose & \\
\hline Akne & $14 \%$ \\
\hline papulöse Dermatose & $2,7 \%$ \\
\hline Hyperpigmentierung & $12,3 \%$ \\
\hline Hypopigmentierung & $4,1 \%$ \\
\hline Lentigines & $2,7 \%$ \\
\hline Lentigo & $2,7 \%$ \\
\hline Feuermal & $2,7 \%$ \\
\hline Melasma & $38,6 \%$ \\
\hline Nävus & $4,1 \%$ \\
\hline Rosacea & $4,1 \%$ \\
\hline faziale Narben & $23,9 \%$ \\
\hline
\end{tabular}

Der Skindex-16 beinhaltet 16 abgefragte Punkte, die auf die Symptomatik (Jucken, Brennen, Schmerz, Irritationen, Erscheinungsbild der Haut, Persistenz), auf Gefühle (Emotionen wie Frustration, Depression etc.) sowie auf das soziale Umfeld eingehen (zwischenmenschliche Beziehungen im täglichen Leben, in der Freizeit, sonstige Aktivitäten und bei der Arbeit). Die Bemessungsskala reicht von 1-7 und die Score-Spannbreite von 16-102. Je höher der Score, umso schlechter die „QOL“ [4]. Der „DLQI“ ist ein einfaches 17 Fragen umfassendes, hauterkrankungsspezifisches Instrument. Hier gilt, je niedriger der Score, desto höher ist die „QOL“ [5]. Der Skindex-16 und der „DLQI“ sind zwei standardisierte Fragebogen zur Bestimmung der „Health-related Quality of Life“" (HRQOL).

Zur Messung des Grades der subjektiv empfundenen Stigmatisierung wird der „FNE“- Fragebogen eingesetzt. Die Bemessungsskala reicht von 1-12 und die Score-Spannbreite von 0-60. Je höher der Score, umso ausgegrenzter erleben sich die Betroffenen.

Die oben angeführten Messverfahren wurden in einer Studie eingesetzt, die unter klinischen Bedingungen in der Wake Forrest Universität der medizinischen Hochschule, Winston Salem, North Carolina unter der Leitung von Dr. Feldmann et al durchgeführt wurde. Ziel der Studie war es herauszufinden, wie stark der Effekt eines Korrektur-Make-ups (Dermablend ${ }^{\circledR}$ ) auf die Lebensqualität der Patienten mit moderaten bis schweren Hautunregelmäßigkeiten im Gesicht ist. Die dreimonatige Untersuchung bezog 73 Frauen im Alter von 18-45 Jahren ein, die unter fazialen Hautveränderungen litten (Vitiligo, Feuermal, Melasma, Narben, Acne, Rosazea etc.) (Tab. 2).

Die Datenerhebung erfolgte zu fünf verschiedenen Zeitpunkten:

1. vor der ersten Anwendung

2. sofort nach der ersten Anwendung

3. nach 15 Tagen

4. nach 1 Monat

5. nach 3 Monaten 


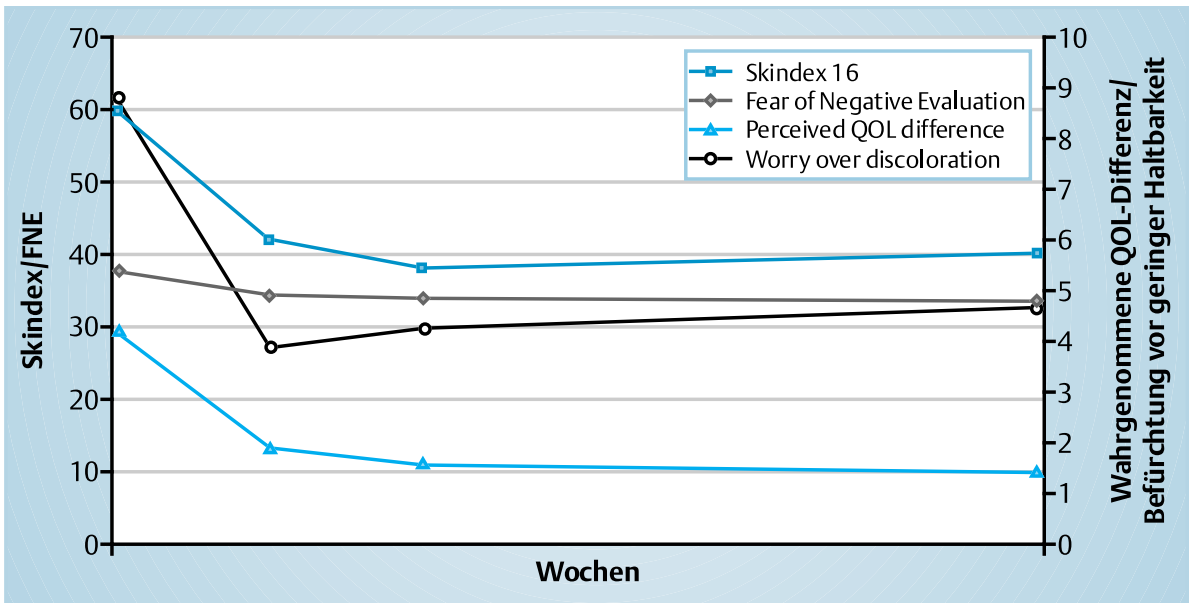

Abb. 1 Untersuchung der Haltbarkeit von Dermablend ${ }^{\circledR}$ unter extremen Bedingungen

Nach drei Monaten zeigte sich ein überzeugendes Ergebnis (Abb. 1):

1. eine Verbesserung des Skindex-16 um $33 \%(p<0.001)$

2. eine Reduktion des „DLQI“ um $45 \%(\mathrm{p}<0.001)$

3. eine „FNE“-Reduktion um $11 \%(\mathrm{p}<0.001)$

Die „HRQOL“ steigt unter Dermablend in statistisch relevanten Größenordnungen an, mit anhaltendem Effekt in jeder Followup-Untersuchung [6].

\section{Fazit}

Faziale Dyschromien und/oder narbige Veränderungen haben einen signifikanten Einfluss auf die „QOL“ Betroffener. Messbare psychologische Charakteristika zeigen diesen Einfluss deutlich auf. Die „QOL“ kann unter Anwendung von korrigierender Kosmetik effektiv verbessert werden. Dermablend stellt hier eine wertvolle Option für die betroffenen Patienten dar, mit dessen Hilfe sich das Körperbild und auch das Selbstwertgefühl verbessern.
Kenntnisse auf dem Gebiet der Camouflage-Technik sind für den Dermatologen eine sinnvolle Ergänzung des Therapiespektrums. Eine fundierte und individuelle Beratung des Patienten durch den Arzt ist sehr wichtig. Der Arzt stellt hier eine kompetente Beratungsinstanz auf diesem Gebiet dar.

\section{Literatur}

${ }^{1}$ Cunliff WJ. Acne and unemployment. Br J Dermatol 1986; 115: 386

${ }^{2}$ Alomar Muntanola A. VICHY-Symposium. Derm-Kompakt, EADV 2003

${ }^{3}$ Finlay AY. Quality of live measurement in dermatology: a practical guide. Br J Dermatol 1997; 136: 305 - 314

${ }^{4}$ Chren $\mathrm{M}$ et al. Skindex, a quality-of-life measure for patients with skin disease: reliability, validity and responsiveness. J Invest dermatol 1996; 107: 707-713

${ }^{5}$ Finlay AY, Khan GK. Dermatology Life Quality Index (DLQI) - a simple practical measure for routine clinical use. Clin Exp Dermatol 1994; 19: $210-216$

${ }^{6}$ Feldmann S et al. VICHY-Symposium. Derm-Kompakt, EADV 2003 